\title{
Prevalence, incidence and stability of premenstrual dysphoric disorder in the community
}

\author{
H.-U. WITTCHEN, ${ }^{1}$ E. BECKER, R. LIEB AND P. KRAUSE \\ From the Technical University of Dresden, Institute of Clinical Psychology and Psychotherapy, Dresden; \\ and Max-Planck Institute of Psychiatry, Munich, Germany
}

\begin{abstract}
Background. Despite an abundance of clinical research on premenstrual and menstrual symptoms, few epidemiological data provide estimates of the prevalence, incidence, co-morbidity, stability and correlates of premenstrual dysphoric disorder (PMDD) in the community.
\end{abstract}

Aims. To describe the prevalence, incidence, 12 co-morbidity factors and correlates of threshold and subthreshold PMDD in a community sample of young women.

Methods. Findings are based on prospective-longitudinal community survey of 1488 women aged 14-24, who were followed-up over a period of 48 months (follow-up, $N=1251$ ) as part of the EDSP sample. Diagnostic assessments were based on the Composite International Diagnostic Interview (CIDI) and its 12-month PMDD diagnostic module administered by clinical interviewers. Diagnoses were calculated using DSM-IV algorithms, but daily ratings of symptoms, as required, were not available.

Results. The baseline 12-month prevalence of DSM-IV PMDD was 5.8\%. Application of the diagnostic exclusion rules with regard to concurrent major depression and dysthymia decreased the rate only slightly $(5 \cdot 3 \%)$. An additional $18.6 \%$ were 'near-threshold' cases, mostly because they failed to meet the mandatory impairment criterion. Over the follow-up period only few new PMDD cases were observed: cumulative lifetime incidence was $7 \cdot 4 \%$. PMDD syndrome was stable across 48 months with $<10 \%$ complete remissions among baseline PMDD cases. The 12-month and lifetime co-morbidity rates were high (anxiety disorders $47.4 \%$, mood disorders $22.9 \%$; somatoform $28.4 \%$ ), only $26.5 \%$ had no other mental disorder. Particularly high odds ratios were found with nicotine dependence and PTSD. In terms of correlates increased rates of 4-weeks impairment days, high use of general health and mental health services, and increased rates of suicide attempts were found.

Conclusion. In this sample of adolescents and young adults, premenstrual symptoms were widespread. However, DSM-IV PMDD was considerably less prevalent. PMDD is a relatively stable and impairing condition, with high rates of health service utilization, increased suicidality and substantial co-morbidity.

\section{INTRODUCTION}

The existence of cyclically recurring premenstrual symptoms of clinical significance has been recognized for decades (Frank, 1931) and has been labelled premenstrual syndrome (PMS).

${ }^{1}$ Address for correspondence: Dr Hans-Ulrich Wittchen, Technical University of Dresden, Institute of Clinical Psychology and Psychotherapy, Chemnitzer Str. 46 D-011187 Dresden, Germany.
Subsequent studies in clinical settings and a few in the community have pointed out that PMS symptoms are widespread, with significant attendant clinical, public health and socio-economic implications (Bergsjo et al. 1975; Woods et al. 1982a, b; Logue \& Moos, 1986). More stringent attempts to define and operationalize diagnostic criteria for severe premenstrual conditions, however, have occurred more recently. The revised 3rd edition of DSM (DSM-III-R, 
APA, 1987) suggested in its appendix for conditions requiring further study, the category of late luteal phase dysphoric disorder (LLPDD) for severe forms of PMS with criteria predominantly characterized by mood symptoms. In DSM-IV (APA, 1994) the diagnosis was retained under the name 'Premenstrual Dysphoric Disorder (PMDD) in this appendix, with more refined and slightly different criteria along with the suggestion to diagnose and code this condition as 'depressive disorders not otherwise specified'. This latter suggestion referred to findings of the DSM-IV mood disorder work group (Hurt et al. 1992) in a clinical sample of 670 women, keeping prospective longitudinal ratings, that supported earlier claims (Halbreich \& Endicott, 1985) that many women will experience a premenstrual depressive syndrome that cross-sectionally mimics an atypical form of major depressive disorder.

Despite an abundance of clinical research on premenstrual and menstrual symptoms, there are only few reliable and comprehensive epidemiological data available that do provide estimates about the prevalence, incidence, risk factors and correlates of both LLPDD and PMDD in the community and in clinical settings. Most of the available epidemiological evidence is confined to numerous studies that used questionnaire data to describe the frequency and the type of PMS symptoms as well as selected correlates in both adolescent (Cleckner-Smith et al. 1998) and adult women (Widholm \& Kantero, 1971; Van Keep \& Lehert, 1981; Andersch et al. 1986; Logue \& Moos, 1986, Boyle et al. 1987; Johnson, 1987; Johnson et al. 1988; Monagle et al. 1993). Disregarding considerable variation in instruments, sampling and design of these studies, there is considerable evidence that the vast majority of women have at least some PMS symptom; however, the prevalence of more severe PMS symptoms seems to be considerably lower; estimates for variably defined severe PMS symptomatology vary between 1 and $9 \%$. As to our current knowledge, there are only two community studies available, which have used stricter epidemiological standards concerning PMS as a syndrome and which provided prevalence estimates for clinically relevant PMS conditions. Results from the Zurich cohort studies (Merikangas et al. 1993; Angst et al. 2001) suggest for their community cohort of 299 women, aged 21 to 35 , that according to their study instrument $8.1 \%$ could be regarded as having severe and $13.6 \%$ as having moderate perimenstrual syndromes. Deuster et al. (1999) completed the, so far, most powerful survey in the community. Using telephone interview survey data incorporating the administration of the Menstrual Distress Questionnaire in a sample of 874 women in Virginia, US, they reported a cross-sectional prevalence of $8 \cdot 3 \%$ (95\% confidence interval $6 \cdot 4-10 \cdot 2 \%$ ) for females aged 18-44. This study is also the only one that examines associations with a number of social and behavioural factors including nutrition, physical exercise, stress and affective state.

Regarding all these studies some general limitations should be highlighted: $(i)$ none of the studies provides prevalence estimates of strictly defined DSM diagnoses of either LLPMD or PMDD; (ii) almost all studies rely on questionnaire data (they neither address specifically and comprehensively DSM symptom criteria, nor do they take into account the diagnostic exclusionary criteria or the difficult assessment criterion of prospective symptom diaries over subsequent menstrual cycles); (iii) to our knowledge only one study examined specifically premenstrual symptoms in adolescents; (iv) furthermore, very few provide additional epidemiological standard measures with regard to incidence, stability as well as social, clinical and behavioural risk factors and correlates.
Aims
Given the lack of such data, this paper reports prevalence and 4-year incidence data for symptoms and the diagnosis of PMDD ac- cording to the criteria of DSM-IV in a general population sample of over 1000 women. It is noteworthy though, that as in all other available epidemiological studies, no prospective daily ratings of PMS symptoms were made as required by the DSM-IV criteria. As part of the Early Developmental Stages of Psychopathology study (EDSP, Wittchen et al. 1998), this prospective community study provides a wider range of information concerning vulnerability and risk factors as well as psychopathological conditions (co-morbidity with other mental disorders) and allows incidence and relative stability of this condition over a period of 4 years to be estimated. 


\section{METHOD}

The EDSP is based on a 4-year prospectivelongitudinal design with up to three assessment points (approximately 20 months apart) in a representative community sample of 3021 adolescents and young adults living in the Munich area. Age of respondents at baseline (T0) was 14 to 24 years. As the study is designed with special interest in early stages of disorders and to increase power, 14- to 15-year-olds were sampled at twice the probability of persons 16 to 21 years-of-age, and 22- to 24-year-olds were sampled at half this probability. Furthermore, this younger cohort (aged 14 to 17 at baseline) was examined twice over the study periods (first follow-up investigation (T1)), whereas baseline 18 to 24-year-olds were reinterviewed only once, as part of the joint final follow-up investigation (T2), approximately 42 months later after the baseline assessment.

\section{Sampling of the respondents and baseline investigation}

The EDSP sample was drawn randomly from the 1994 government population registers of residents in metropolitan Munich and the surrounding countries with an expected age range for the sampled subjects between 14 and 24 (birth cohorts 1970-1981) at the time of the baseline interview in 1995. A total of 3021 interviews were completed at baseline, resulting in a response rate of $71 \%$. Detailed descriptions of the sampling procedures of the baseline investigation and reasons for non-response have already been presented elsewhere (Wittchen et al. 1998; Lieb et al. 2000).

For the purpose of this paper, only female respondents of this survey were analysed $(N=$ 1488); response rates for females at baseline (T0) were $69 \cdot 7 \%$. The most frequent reasons for not participating were refusal $(18.6 \%)$ or lack of time $(3 \%)$. The conditional response rate was $86.8 \%$ at the first follow-up (T1) and $84.7 \%$ at the (T2) second follow-up. Most frequent reasons for not participating at the subsequent follow-up investigations were refusal $(6.6 \%$ and $9.3 \%)$, not locatable, or moved abroad $(3.1 \%$ and $1.8 \%$ ). The age range of the sample at the final follow-up was 18 to 29. Due to the large number of respondents, it was technically not feasible to keep a constant time interval between the different waves. The first follow-up was conducted in 1996/1997, an average of $19 \cdot 7$ months after baseline (range: 14 to 25 months). This wave included only the respondents who were 14 to 17 years-of-age at baseline $(N=$ 1395; birth cohorts 1977-1981). The second follow-up investigation included all baseline respondents and was conducted in 1998/1999, an average of 42 months after the baseline investigation (range: 34 to 50 months).

\section{Baseline and follow-up sociodemographic characteristics}

Due to the requirement of stable menstruation patterns, only $1091(73.3 \%)$ of the total of 1488 female participants at baseline completed the PMS assessment. At $\mathrm{T} 1$ among those aged 14-17 at baseline, $N=586$ out of the total of 591 females $(99 \cdot 2 \%)$ completed the PMS assessment, while at $\mathrm{T} 2$ among all baseline participants recontacted, $N=1233$ out of 1251 (98.6\%) completed the PMS assessment.

The sociodemographic characteristics of the study sample was already described elsewhere in more detail (Wittchen et al. 1998; Lieb et al. 2000). Briefly, at baseline, $35.5 \%$ of the respondents were in secondary school; among these the majority attended Gymnasium (secondary education between the ages of 10 to 19 that prepares students for entrance to university), $25.6 \%$ of the respondents attended university, $9.9 \%$ were in job training, $22.9 \%$ were employed and $0.9 \%$ were currently unemployed. The majority of respondents $(57.6 \%)$ were still living with their parents and only a few $(4.9 \%)$ were married. The vast majority of respondents were classified as middle class $(60 \cdot 8 \%)$ and $6.6 \%$ were grouped as lower social classes, both of which proportions are consistent with the demographics of the Munich population. Almost all respondents indicated having had their first menstrual period, the majority prior to age 14 .

At the time of the second follow-up investigation, only a few $(12.6 \%)$ were still attending a school other than university $(25 \%)$, while more respondents $(37.3 \%)$ were employed. A lower proportion of respondents $(35.2 \%)$ still lived with their parents, while more subjects lived with a partner $(28.7 \%)$. No remarkable changes were found with regard to the respondents, reported social class and financial situation. The 
proportion of married respondents doubled from baseline $(4.9 \%)$ to second follow-up $(10 \cdot 3 \%)$. The majority $(45 \cdot 8 \%)$ indicated the use of contraceptives in the 12-month period preceding the T2 assessment.

\section{Assessment of adolescents and young adults}

In all three waves of investigation, symptom and diagnostic assessment were based on the computer assisted version of the Munich-Composite International Diagnostic Interview (DIA-X/MCIDI; Wittchen \& Pfister, 1997). The M-CIDI is an updated version of the World Health Organization's CIDI version 1.2 (WHO, 1990) incorporating questions assessing DSM-IV and ICD-10 criteria. The M-CIDI allows for the standardized assessment of symptoms, syndromes and diagnoses of a wide range of DSMIV substance use and mental disorders (and additional various subtypes of main disorders) along with information about onset, duration, clinical and psychosocial severity. ${ }^{1}$ Detailed descriptions of M-CIDI along with its assessment procedures and psychometrics have already been described in detail elsewhere (Wittchen, 1994; Reed et al. 1998; Wittchen et al. 1998). In the baseline investigation the lifetime version of the M-CIDI was used to assess lifetime and 12-month information. For the two follow-up investigations, the M-CIDI was modified to cover the 12-month period prior to the follow-up interview as well as the remaining interval between the investigations (12-monthinterval-version). Diagnostic assignment is based on the M-CIDI diagnostic package 'DSM-IV diagnostic algorithms' (Wittchen \& Pfister, 1997). Due to the standardization and computerization of the interview and assignment of diagnoses, the assessment can be regarded as highly objective. In each wave, the respondents were given a special M-CIDI response booklet with several symptom lists and visual aids to improve lifetime recall, facilitate memory search, and help the respondent to answer onset and recency questions of reported symptoms. Furthermore, the response booklet contained several scales and questionnaires to assess selected psychological constructs.

\footnotetext{
${ }^{1}$ The complete M-CIDI is available from the authors on request.
}

\section{M-CIDI-PMDD module and DSM-IV \\ criteria}

In addition to the information concerning the respondents' menstruation history, anthropometric information as well as contraceptive use, each wave included an identical assessment of premenstrual syndromes according to criteria of DSM-IV PMDD. The PMDD module was administered only in females with at least 3 years history of regular menstruation and consisted of a series of questions pertaining to the past 12 months: (a) to assess DSM-IV criterion A, requires five or more symptoms (i.e. markedly depressed mood, marked anxiety, tension, marked affective lability, persistent anger or irritability) out of a total of 11 symptoms in most menstrual cycles during the past 12 months, separate questions evaluated the presence of DSM-IV symptom criteria during the past 12 months, respectively their presence during the majority of all menstrual cycles in the past 12 months; (b) supplemented by one question to ascertain whether these symptoms occurred consistently in the week before onset of menstruation and stopped in the week post-menses (A); (c) three questions were used to evaluate criterion B for impairment and psychosocial interference. Criterion $\mathrm{C}$ (the disturbance is not merely an exacerbation of symptoms of another disorder, such as major depression, or panic disorder) was assessed whenever the person fulfilled during the same time period criteria of both another disorder as well as PMDD. Criterion $\mathrm{D}$, requiring prospective daily ratings during at least two consecutive menstrual cycles was not feasible, therefore in accordance with DSM-IV PMDD diagnoses in this paper should be regarded as 'provisional' (prospective ratings).

\section{Reliability and validity of the M-CIDI}

Reliability and procedural validity of the MCIDI were tested in several studies. Briefly, test-retest reliability (retest after an average period of 39 days, $N=60$ of the community sample) was fair to good for substance use disorders, ranging from kappa $(\kappa)=0.64$ (Yule's $\mathrm{Y}=0.80)$ for drug use disorders to $\kappa=0.78$ (Yule's $\mathrm{Y}=0.82$ ) for alcohol use disorders (Wittchen et al. 1998). Procedural clinical val- 
idity was examined in 68 patients comparing MCIDI diagnoses with clinicians' best-estimate diagnoses and taking into account all available information (medical records, diagnostic interview, diagnostic checklist) at the end of the patients' treatment (Reed et al. 1998). Detailed analyses covering validity and reliability of single diagnostic criteria and age, frequency, and quantity information are provided elsewhere (Wittchen, 1994; Reed et al. 1998; Wittchen et al. 1998).

\section{Interviewer and interviewer training}

In all assessment stages interviews were conducted by a total of 57 clinical interviewers, mostly graduate psychologists. In addition, 25 professional health research interviewers recruited from a survey company (Infratest Gesundheitsforschung) were involved in the baseline investigation. All interviewers participated in at least 1 week of training for both the computerized and the paper-pencil version of the M-CIDI and several follow-up trainings throughout each wave as well as at the beginning of each additional wave. This was followed by at least ten practice interviews that were closely monitored and supervised by the staff of the study. For a more comprehensive overview about field work methodology see Lieb et al. 2000 .

\section{Sample and weighting}

As the EDSP is designed with special interest in early stages of psychopathology, 14- to 15-yearolds were sampled at twice the probability of 16to 21-year-olds, and 22- to 24-year-olds were sampled at half this probability (see Wittchen et al. 1998). This sampling strategy allows particularly precise estimations of measures used for comparative analyses for the age group of primary interest, i.e. 14- to 15-year-olds. Due to the different sampling probabilities, relative weights inversely proportional to the sampling fraction are used in the estimation of measures which are generalized to the sampling frame. In addition, these weights also account for nonresponse according to age, gender and geographic distribution (urban $v$. rural) of the respondents to that of the registered sampling frame. For data from the first follow-up investigation which include only the 14- to 17 -year- olds, a special weight was computed for the subpopulation of the younger cohort, also adjusting for drop-out from baseline to first follow-up according to age, gender, geographic distribution and non-response. For data from the second follow-up, the same weights as those of the baseline investigation were used, because there was no selective attrition due to age, gender, or geographic distribution for which we needed to adjust.

\section{RESULTS}

\section{Prevalence of PMDD at baseline among 14 to} 24-year-olds and cumulative incidence

According to the M-CIDI DSM-IV criteria and algorithms, irrespective of age group examined, the weighted estimated 12-months prevalence of PMDD at baseline was $5.8 \%$. There was a moderate increase (Table 1) of new cases (unaffected at T0) at the final follow-up of $2 \cdot 3 \%$ among the previously 14 to 17 -year-olds and $2.6 \%$ among those aged initially $18-24$. The additional $\mathrm{T} 1$ assessment in the younger cohort suggested a fairly equal incidence increase from $\mathrm{T} 0$ to $\mathrm{T} 1$ and $\mathrm{T} 1$ to $\mathrm{T} 2$, respectively. The resulting total overall cumulative incidence for PMDD according to M-CIDI-DSM-IV algorithms across all assessments up to age 29 is therefore $7 \cdot 4 \%$ (raw $N: 104, N w: 112$ cases). We also examined among baseline and cumulative incidence cases the effect of the strict application of diagnostic exclusion criteria of DSM-IV with regard to major depression and dysthymia, panic disorder, GAD as well as full somatoform disorders. Taking current major depression and dysthymia diagnoses into account, the baseline rate would drop marginally from $5.8 \%$ to $5 \cdot 3 \%$, the cumulative incident rate from $7.5 \%$ to $6.7 \%$. Taking the other disorders into account there was an additional drop of $0 \cdot 2-0 \cdot 5 \%$.

\section{Prevalence of premenstrual symptoms in the} total sample, threshold and subthreshold cases

In addition to the PMDD threshold cases, $18.6 \%$ of the sample met almost all diagnostic criteria and were thus classified as subthreshold PMDD in our study. Prevalence estimates for full and subthreshold PMDD were identical in females aged 14-17 years and 18-24 years of age at baseline. Among subthreshold cases the lack 
Table 1. Prevalence of PMDD at baseline among 14-24-year-olds and 40 months cumulative incidence

\begin{tabular}{|c|c|c|c|c|c|c|c|c|c|}
\hline & \multicolumn{9}{|c|}{ 12-months ( $\%$ weighted) estimates at each assessment and overall cumulative incidence $\mathrm{T} 0 / \mathrm{T} 1 / \mathrm{T} 2$} \\
\hline & \multicolumn{3}{|c|}{$12 \operatorname{mos} \mathrm{T} 0$} & \multicolumn{3}{|c|}{ Incident cases $\mathrm{T} 2$} & \multicolumn{3}{|c|}{ Cum. incidence $\mathrm{T} 0-\mathrm{T} 2$} \\
\hline & $\mathrm{Nw}$ & $\% \mathrm{w}$ & $95 \% \mathrm{CI}$ & $N \mathrm{w}$ & $\% \mathrm{w}$ & $95 \% \mathrm{CI}$ & $N \mathrm{w}$ & $\% \mathrm{w}$ & $95 \% \mathrm{CI}$ \\
\hline \multicolumn{10}{|c|}{ Group by age at $\mathrm{T} 0$} \\
\hline $14-17$ & 13 & $5 \cdot 8$ & $3 \cdot 5-9 \cdot 3$ & 8 & $2 \cdot 3$ & $1 \cdot 3-4 \cdot 3$ & 30 & $6 \cdot 7$ & $4 \cdot 9-9 \cdot 0$ \\
\hline $18-24$ & 61 & $5 \cdot 8$ & $4 \cdot 2-7 \cdot 8$ & 22 & $2 \cdot 6$ & $1 \cdot 6-4 \cdot 2$ & 83 & $7 \cdot 7$ & $6 \cdot 0-10 \cdot 0$ \\
\hline Total & 74 & $5 \cdot 8$ & $4 \cdot 4-7 \cdot 5$ & 30 & $2 \cdot 5$ & $1 \cdot 7-3 \cdot 7$ & 113 & $7 \cdot 4$ & $6 \cdot 0-9 \cdot 1$ \\
\hline
\end{tabular}

of persistent impairment associated with premenstrual symptoms was by far $(72 \%)$ the most frequent reason for not meeting criteria for full diagnosis.

At baseline only $20 \cdot 2 \%$, irrespective of age (design-based $F(1,1090)=0.0693 ; \quad P=0.792$ ) did not endorse any of the 11 symptoms questions. Among the 79.8\% endorsing at least one PMS symptom as having been present frequently in the past 12 months, $55.3 \%$ reported having had the symptom(s) in almost all premenstrual episodes: $16.3 \%$ reported just one; $15.9 \%$ two; $11.9 \%$ three; $10.9 \%$ four; and $24.8 \%$ five or more. The cumulative incidence rate of PMS symptoms over all three waves was only slightly higher. In the total sample the five most frequently endorsed premenstrual symptoms at baseline and over all three waves were: physical complaints (T0: 44.9\%), affect lability $(41 \cdot 7 \%)$, fatigability $(36.6 \%)$, depressed mood $(31 \cdot 4 \%)$ and appetite/craving $(30 \cdot 3 \%)$.

The rank order of PMDD items among those with a DSM-IV threshold diagnosis PMDD was : depressed mood, hopelessness/self-depreciation $(90.5 \%)$ ranked first, followed by affect lability $(89.7 \%)$, irritability/anger $(81.5 \%)$, fatigability $(78.6 \%)$ and physical complaints $(78 \cdot 1 \%)$, anxiety/tension $(67 \%)$, and decreased interest $(63 \cdot 3 \%)$ (Fig. 1a). The comparison with subthreshold cases (Fig. $1 b$ ) reveals a similar spectrum of symptoms, however, with markedly lower frequencies for the following symptoms: decreased interest (threshold, $74 \%$ v. 48.7\%; among subthresholds OR 3.0, 95\% CI 1.7-5.2), depressed mood $(93.5 \%$ v. $83.9 \%$; OR $2 \cdot 8$, $P<0.05 ; 95 \%$ CI 1.1-6.9), irritability/anger $(86.9 \% \vee 70.6 \%,=$ OR $2.8, P<0.05 ; 95 \%$ CI 1.4-5.7), feeling overwhelmed (49\% v. 28.3\%;
OR $2 \cdot 4, P<0 \cdot 05 ; 95 \%$ CI $1 \cdot 5-4 \cdot 1)$ and difficulty in concentrating $(54 \cdot 1$ v. $37 \cdot 7 \%$; OR $2 \cdot 0$, $P<0.05 ; 95 \%$ CI $1 \cdot 2-3 \cdot 2)$.

\section{Stability of PMDD diagnostic status over the assessment period}

Fig. 2 cross-tabulates baseline diagnostic status $(\mathrm{PMDD}=$ all DSM-IV criteria are fulfilled, subthreshold $=$ just one of diagnostic criteria is not met, symptomatic = having at least two PMS symptoms, no PMDD = no PMS symptoms) with the diagnostic status at followup (T1, T2). While only few subjects with no PMDD at baseline had at least subthreshold PMDD symptoms at the first or second followup (proportion: $4 \cdot 6 \%$ ), $46.7 \%$ of those with baseline subthreshold PMDD symptoms and $48 \%$ among those with baseline threshold PMDD were at least near threshold cases at T1 or T2. The respective proportions for full remission were $6.7 \%$ and $4.9 \%$ respectively.

\section{Patterns of 12-month co-morbidity}

Table 2 reports the frequency of mental disorders and their co-morbidity rates among non-PMDD cases in comparison with threshold and subthreshold PMDD cases with their odds ratios and significance level controlled for by age. At baseline and in comparison to non-PMDD cases $(57 \cdot 2 \%$ with no disorder), only $26 \cdot 5 \%$ of all PMDD threshold cases and $39.8 \%$ of all subthreshold cases had no other mental disorders. Thus, threshold (OR 3·7, $95 \%$ CI 1·9-7·0) and subthreshold PMDD cases (OR 2.0; $95 \%$ CI 1.4-2.8) were significantly more likely to have at least one mental disorder and were also more likely to have at least three 12-month diagnoses of mental disorders (threshold PMDD, OR 7.3; 
(a)



(b)

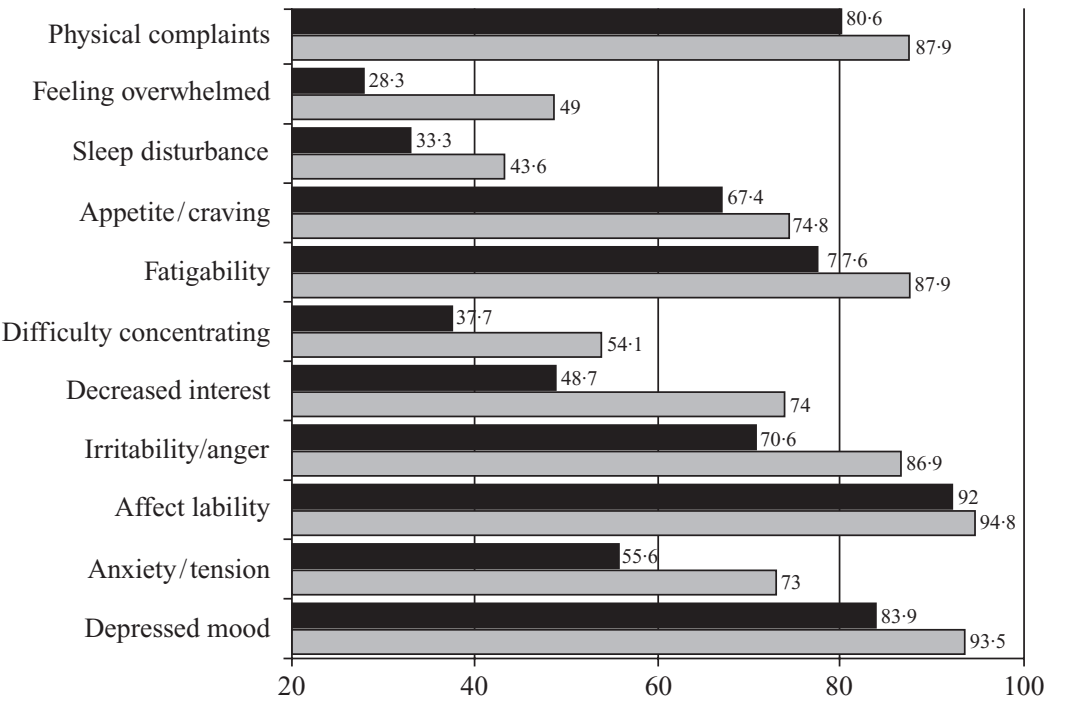

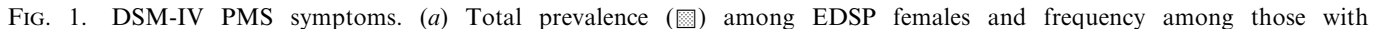
PMDD diagnosis ( $\square$ ). (b) Cumulative incidence over 4 years among females, threshold ( $\square, N=104$ ) and subthreshold PMDD $(\mathbf{\square}, N=321)$

95\% CI 3.8-13.9; subthreshold, $2 \cdot 7 ; 95 \%$ CI $1 \cdot 7-4 \cdot 4)$. It is noteworthy - as stated earlier that symptoms for example for somatoform disorders and PMDD were assessed separately, thus no 'double counting' of caseness occurred.

Most frequent significant associations were with: (a) anxiety disorders (47.4\% PMDD versus $22.5 \%$ among non-PMDD cases (odds ratio $3 \cdot 1$
(95\% CI 1.7-5.5)), due to associations with social phobia (OR 4.2 (2.0-8.8) and simple phobia (OR $3 \cdot 3(1 \cdot 7-6 \cdot 3)$, particularly of blood injury and situational type; $(b)$ all mood disorders (OR 3.0 (1.6-5.7), including bipolar I and II; (c) PTSD (11.7 (3.0-46.2); (d) somatoform disorders (pain, and SSI-4/6, OR 3.4 (1.8-6.6); and $(e)$ nicotine dependence (OR $3 \cdot 4 ; 1 \cdot 8-6 \cdot 3$ ). Subthreshold PMDD associations with other 


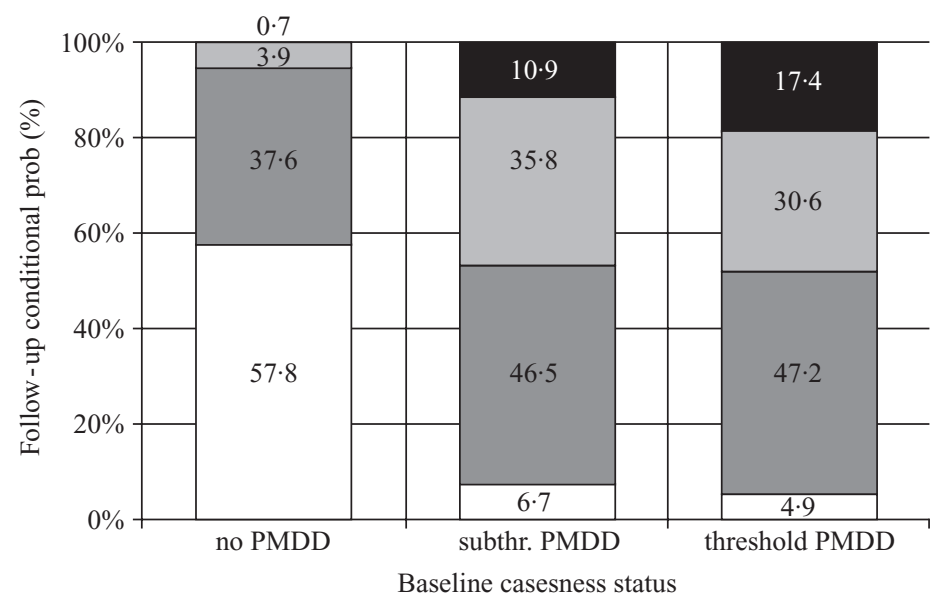

FIG. 2. Conditional probability of baseline PMDD caseness status at 40 months follow-up ( $\square$, threshold PMDD; , subthreshold PMDD; $\square$, symptomatic; $\square$, no PMDD).

Table 2. Twelve months co-morbidity among threshold and subthreshold PMDD cases at baseline $(T 0)$

\begin{tabular}{|c|c|c|c|c|c|}
\hline & \multirow{2}{*}{$\begin{array}{l}\text { No PMDD } \\
\frac{(N=828)}{\% \mathrm{~W}}\end{array}$} & \multicolumn{2}{|c|}{$\begin{array}{l}\text { PMDD } \\
(N=74)\end{array}$} & \multicolumn{2}{|c|}{$\begin{array}{l}\text { Subthreshold PMDD } \\
\qquad(N=201)\end{array}$} \\
\hline & & $\% \mathrm{w}$ & OR & $\% \mathrm{w}$ & OR \\
\hline Alcohol abuse/dep. & 4.9 & $10 \cdot 2$ & $2 \cdot 2$ & $6 \cdot 7$ & $1 \cdot 4$ \\
\hline Drug abuse/dep. & $1 \cdot 0$ & $2 \cdot 1$ & $2 \cdot 2$ & $3 \cdot 1$ & $3 \cdot 3^{*}$ \\
\hline Nicotine dep. & $14 \cdot 6$ & $36 \cdot 8$ & $3 \cdot 4 * * *$ & $21 \cdot 1$ & $1 \cdot 6^{*}$ \\
\hline Any affective disorder & $12 \cdot 3$ & $29 \cdot 8$ & $3 \cdot 0^{* *}$ & $21 \cdot 0$ & $1 \cdot 9^{*}$ \\
\hline Bipolar I & $0 \cdot 8$ & $5 \cdot 7$ & $7 \cdot 9 * *$ & $3 \cdot 8$ & $5 \cdot 3^{*}$ \\
\hline Bipolar II & 0.6 & $4 \cdot 9$ & $8 \cdot 1^{* *}$ & $0 \cdot 3$ & 0.5 \\
\hline MDD-single & $4 \cdot 3$ & $11 \cdot 8$ & $3 \cdot 0^{*}$ & $6 \cdot 0$ & $1 \cdot 4$ \\
\hline MDD-recurrent & $2 \cdot 6$ & $4 \cdot 2$ & 1.6 & $3 \cdot 5$ & $1 \cdot 3$ \\
\hline Dysthymia & 3.9 & $9 \cdot 5$ & $2 \cdot 6^{*}$ & $34 \cdot 6$ & $1 \cdot 8^{*}$ \\
\hline Any anxiety disorder & $22 \cdot 5$ & $47 \cdot 4$ & $3 \cdot 1 * * *$ & $10 \cdot 4$ & $3 \cdot 9^{*}$ \\
\hline Panic attacks & $2 \cdot 9$ & $5 \cdot 8$ & $2 \cdot 1$ & $5 \cdot 2$ & $3 \cdot 7 *$ \\
\hline Panic disorder & $1 \cdot 4$ & 2.5 & $1 \cdot 8$ & $2 \cdot 7$ & 1.7 \\
\hline Agoraphobia & $1 \cdot 6$ & $5 \cdot 2$ & $3 \cdot 4$ & $5 \cdot 6$ & $1 \cdot 5$ \\
\hline Social phobia & $6 \cdot 0$ & $21 \cdot 1$ & $4 \cdot 2^{* * *}$ & $10 \cdot 6$ & $1 \cdot 8^{*}$ \\
\hline Specific phobia & $12 \cdot 5$ & $31 \cdot 7$ & $3 \cdot 3^{* * *}$ & $19 \cdot 6$ & $1.7^{*}$ \\
\hline Phobia NOS & $3 \cdot 7$ & $6 \cdot 9$ & 1.9 & $5 \cdot 6$ & 1.5 \\
\hline GAD & $2 \cdot 6$ & $1 \cdot 3$ & 0.5 & $4 \cdot 2$ & $1 \cdot 6$ \\
\hline $\mathrm{OCD}$ & 0.5 & $1 \cdot 4$ & $3 \cdot 1$ & $2 \cdot 2$ & $4 \cdot 9 *$ \\
\hline PTSD & 0.8 & $8 \cdot 3$ & $11 \cdot 7 * * *$ & $1 \cdot 9$ & $2 \cdot 5$ \\
\hline Any somatoform disorder & $10 \cdot 4$ & $28 \cdot 4$ & $3 \cdot 4 * * *$ & $12 \cdot 4$ & $1 \cdot 2$ \\
\hline SSI4/6, somatization & 1.8 & $9 \cdot 7$ & $5 \cdot 8 * * *$ & $2 \cdot 7$ & $1 \cdot 5$ \\
\hline Pain & $1 \cdot 2$ & $4 \cdot 9$ & $4 \cdot 3^{*}$ & $2 \cdot 5$ & $2 \cdot 0$ \\
\hline Any eating disorder & $2 \cdot 1$ & $5 \cdot 2$ & $2 \cdot 6$ & $5 \cdot 5$ & $2 \cdot 7 *$ \\
\hline \multicolumn{6}{|l|}{ Co-morbidity } \\
\hline None & $57 \cdot 2$ & $26 \cdot 5$ & $0 \cdot 3^{*}$ & $39 \cdot 8$ & $0 \cdot 5^{*}$ \\
\hline Any & $42 \cdot 8$ & $3 \cdot 5$ & $3 \cdot 7 * * *$ & $60 \cdot 2$ & $2 \cdot 0 * * *$ \\
\hline 1 & $24 \cdot 3$ & $24 \cdot 0$ & $1 \cdot 0$ & $28 \cdot 6$ & $1 \cdot 2$ \\
\hline 2 & $11 \cdot 2$ & $12 \cdot 8$ & $1 \cdot 2$ & $13 \cdot 8$ & $1 \cdot 3$ \\
\hline$\geqslant 3$ & $7 \cdot 3$ & $36 \cdot 8$ & $7 \cdot 3 *$ & $17 \cdot 7$ & $2 \cdot 7 * * *$ \\
\hline
\end{tabular}

Odds ratio (OR) were controlled for by age.

In the co-morbid counts the following groups of DSM-IV diagnoses were considered: any alcohol disorder; any drug disorder; nicotine dependence; any affective disorder; panic disorder; agoraphobia; phobia NOS; social phobia; specific phobia; generalized anxiety disorder (GAD); obsessive-compulsive disorder (OCD); any somatoform disorder; any eating disorder (anorexia, bulimia, binge eating disorder).

* $P<0.05 ; * * P<0.01 ; * * * P<0.001$. 
Table 3. Correlates of threshold and subthreshold PMDD at follow-up (T2)

\begin{tabular}{|c|c|c|c|c|c|}
\hline \multirow[b]{2}{*}{ Indicator } & \multirow{2}{*}{$\begin{array}{c}\begin{array}{c}\text { Non-PMDD } \\
(N=1007)\end{array} \\
\% \mathrm{w}\end{array}$} & \multicolumn{2}{|c|}{$\begin{array}{l}\text { PMDD } \\
(N=87)\end{array}$} & \multicolumn{2}{|c|}{$\begin{array}{l}\text { Subthr. PMDD } \\
\quad(N=189)\end{array}$} \\
\hline & & $\% \mathrm{~W}$ & $\mathrm{OR} \dagger(95 \% \mathrm{CI})$ & $\% \mathrm{~W}$ & $\mathrm{OR} \dagger(95 \% \mathrm{CI})$ \\
\hline 4 wks impairment & $7 \cdot 5$ & $30 \cdot 5$ & $5 \cdot 4 * * *(2 \cdot 3-12 \cdot 3)$ & $13 \cdot 3$ & $2 \cdot 3 * * *(1 \cdot 5-3 \cdot 5)$ \\
\hline Impairment days/past mo (mean) $\$$ & $0 \cdot 4$ & $2 \cdot 0$ & $5 \cdot 1 *(2 \cdot 4-11 \cdot 0)$ & $0 \cdot 4$ & $1 \cdot 1(0 \cdot 6-2 \cdot 0)$ \\
\hline 4 wks disability & $1 \cdot 7$ & $4 \cdot 7$ & $2 \cdot 9(0 \cdot 5-15 \cdot 6)$ & $1 \cdot 1$ & $0 \cdot 6(0 \cdot 1-2 \cdot 9)$ \\
\hline Disability days/past mo (mean) & $0 \cdot 1$ & $0 \cdot 1$ & $1 \cdot 6(0 \cdot 4-7 \cdot 6)$ & 0 & \\
\hline Any mental health treatment & $10 \cdot 2$ & $28 \cdot 3$ & $3 \cdot 4 * *(1 \cdot 5-8 \cdot 0)$ & $20 \cdot 3$ & $2 \cdot 2 * *(1 \cdot 4-3 \cdot 5)$ \\
\hline$\geqslant 3 \mathrm{GP}$ visits $/ 12 \mathrm{mos}$ & $21 \cdot 9$ & $36 \cdot 9$ & $2 \cdot 1 *(1 \cdot 01-4 \cdot 7)$ & $31 \cdot 4$ & $1 \cdot 7 * *(1 \cdot 2-2 \cdot 5)$ \\
\hline$\geqslant 3$ visits and specialist $/ 12 \mathrm{mos}$ & $4 \cdot 5$ & $16 \cdot 9$ & $4 \cdot 3 * *(1 \cdot 4-12 \cdot 8)$ & $5 \cdot 3$ & $2 \cdot 0 *(1 \cdot 0-3 \cdot 9)$ \\
\hline Use of psychotropics & $1 \cdot 5$ & 1.5 & $1 \cdot 0$ & $2 \cdot 1$ & $1 \cdot 3(0 \cdot 4-5 \cdot 1)$ \\
\hline Use of vitamins/minerals & $13 \cdot 7$ & $11 \cdot 7$ & $0 \cdot 8(0 \cdot 2-3 \cdot 1)$ & $13 \cdot 3$ & $0 \cdot 9(0 \cdot 5-1 \cdot 6)$ \\
\hline Suicidal ideation & $4 \cdot 0$ & $8 \cdot 8$ & $2 \cdot 3(0 \cdot 5-11 \cdot 0)$ & $6 \cdot 6$ & $1 \cdot 7(0 \cdot 8-3 \cdot 5)$ \\
\hline Suicide attempts & $3 \cdot 2$ & $15 \cdot 8$ & $4 \cdot 4 * * *(2 \cdot 0-9 \cdot 7)$ & $5 \cdot 0$ & $1.6(0 \cdot 7-3 \cdot 6)$ \\
\hline \multicolumn{6}{|l|}{ Subjective health rating } \\
\hline Poor & $10 \cdot 7$ & $26 \cdot 3$ & $3 \cdot 8 * * *(1 \cdot 6-8 \cdot 9)$ & $22 \cdot 3$ & $2 \cdot 0 *(1 \cdot 2-3 \cdot 6)$ \\
\hline Very bad & $1 \cdot 2$ & $8 \cdot 4$ & $13 \cdot 0 *(1 \cdot 5-16 \cdot 6)$ & $6 \cdot 4$ & $2 \cdot 4(0 \cdot 4-13 \cdot 4)$ \\
\hline
\end{tabular}

$\dagger$ Odds ratios were controlled for age.

Numbers are expressed as percentages unless otherwise stated.

* Impairment and disability days were indicated by their mean and the incidence rate ratio (IRR, calculated with negative binomial regression) was indicated, instead of the odds ratio.

* $P<0.05$; ** $P<0.01$; *** $P<0.001$.

mental disorders appear to be relatively similar, however the associations are generally weaker. The lack of significant associations with PTSD on the one hand, and the significant associations with drug/medication abuse or dependence among subthreshold cases as well as OCD are noteworthy. The examination of 12-month associations at follow-up (T2) revealed an almost identical picture.

We further examined whether baseline PMDD were more likely than non-PMDD cases to develop incident anxiety, affective, or somatoform disorders over the follow-up period. As compared to non-PMDD cases, PMDD cases were less likely to develop new anxiety disorders (incidence rate: $7.6 \%$ v. $13.0 \%$ ). But, they were significantly more likely to develop new depressive episodes $(24.7 \%$ v. $9 \cdot 7 \%$, OR $3 \cdot 1$ $(1 \cdot 4-7 \cdot 1)$ disorders, largely due to an increase in recurrent episodes (single depressive episode $11.2 \%$ (T0) v. $6.0 \%$, recurrent, $4.2 \%$ (T0) $v$. $19 \%$ (T2) as well as somatoform disorders (54.7\% v. $30.9 \%$, OR $2 \cdot 7, P<0.05 ; 95 \%$ CI $1 \cdot 3-5 \cdot 7)$.

\section{Correlates and outcomes}

Table 3 reports some selected correlates of subjects with threshold or subthreshold PMDD at follow-up. Taking into account that PMDD cases endorsed that their disturbance has been associated with significant interference in social role performance during the majority of cycles in the past year, it is noteworthy that only $30.5 \%$ of PMDD cases as compared to $7.5 \%$ of non-PMDD cases reported acute impairments in their professional and everyday activities during the past 4 weeks (OR $5.4 ; 95 \%$ CI $2 \cdot 3-12 \cdot 3)$. The average number of impairment or disability days per month was 2.6 among full PMDD and 1.7 among subthreshold cases.

Both PMDD groups were found to be high utilizers of mental health practitioners, general health as well as other specialist (gynaecologists) services in the past 12 months: $28.3 \%$ of the threshold and $20.3 \%$ of subthreshold PMDD cases reported having been in treatment or in contact with predominantly psychological or psychotherapeutic services. Also, 36.9\% and $31.8 \%$, respectively, had consulted their primary care doctor more than four times during the previous 12 months (OR 2.1; $95 \%$ CI 1.01-4.7); contact rates to specialists doctors were particularly higher in the PMDD group (OR 4.3; $95 \%$ CI $1.4-12.8)$ as compared to the subthreshold group. However, no excess rates of either psychotropic medication use (sedatives, hypnotics, antidepressives) or the current use of over the counter medicines for vitamins, minerals 
and tonics were found in comparison to the control group.

There is a remarkably high risk of suicide attempts among threshold PMDD cases only (15.8\%; OR 5.7; $95 \%$ CI $1.8-18.3)$, but only moderately increased risks of suicidal ideation. There is also a significantly increased risk among both PMDD groups to report a 'poor' or 'very bad' subjective health state.

\section{DISCUSSION}

This is, to our knowledge, the first epidemiological study on PMDD using the DSM-IV criteria in a large community study, based on standardized diagnostic interviews, instead of PMS questionnaires, that also included a wider range of mental disorder. Further strengths of the study are its prospective-longitudinal design allowing the determination of incidence rates and measures of stability as well as of comorbidity rates with other mental disorders and correlates. Among the limitations of the study, one should keep in mind that in diagnosing PMDD, we did not take into account all of the diagnostic exclusions, except for major depression and dysthymia, that had only a marginal effect on prevalence estimates. We were also unable to fulfil DSM-IVs requirement of using prospective diaries across at least two menstrual cycles. A further important limitation of the study is that we examined exclusively PMDD cases aged 14-24 at baseline and followed them up over a period of 48 months. Thus, the results refer to adolescents and young adults and not to women above this age cut-off, for which a second high incidence period after age of 35 has been suggested in the previous literature.

\section{Premenstrual symptoms are widespread}

Using a slightly different symptom list from previous PMS-related studies, tailored strictly according to DSM-IVs mandatory symptom criteria, we first confirmed the findings of previous studies (Widholm \& Kantero, 1971; Van Keep \& Lehert 1981; Andersch et al. 1986; Logue \& Moos, 1986; Boyle et al. 1987; Johnson 1987; Johnson et al. 1988; Monagle et al. 1993; Cleckner-Smith et al. 1998) that PMS symptoms in general and particularly among adolescents are widespread. In each of the assessment waves more than two-thirds of the female sample reported at least one PMS symptom being present during most the menstrual cycles in the past year, and the high proportion of $35 \cdot 3 \%$ of women reporting four or more symptoms and being quite near to DSM-IVs mandatory threshold count of five or more symptoms is indeed remarkable. Taking the CIDI's convention to classify subthreshold disorders by assigning the diagnosis whenever persons fall short of just one of the mandatory criteria, subthreshold PMDD was diagnosed in $18.6 \%$ of the sample. The most frequent reason for not meeting the full diagnostic criterion was the impairment criteria ( $72 \%$ of all subthreshold cases, and neither the symptom count nor the persistence criterion of DSM-IV). The substantial size of this subthreshold group underlines that consistent premenstrual problems are quite frequent phenomena of female adolescents and young adults. It should be mentioned that such young patients are rarely reported in studies from specialist services, where actually few women under age of 25 are obviously seen (Yonkers, 1997).

We were unable to determine in this study, whether our finding of relatively high prevalences of full DSM-IV PMDD among young women reflects the weakness of our study of not having available prospective daily ratings in making the diagnostic decision, or whether this finding reflects the fact, that it usually takes several years before PMDD patients come to treatment. It might also be possible, referring to our finding that only slightly more than onethird of PMDD cases have acute impairments, that these younger sufferers have a considerably milder symptomatology, than treatment samples. However, in interpreting these relatively low impairments one has to consider in epidemiological surveys of conditions of a cyclic nature, that actually very few cases were in an acute phase of their illness at the time of the interview.

\section{PMDD as a full-blown disorder is considerably less frequent}

As already suggested in the majority of previous publications, PMDD seems to be considerably less frequent. We estimated a 12-month baseline 
prevalence rate of $5.4 \%$ (95\% CI $4.4-7.5 \%)$ among 14-24-year-olds. Despite considerable design and instrumental differences, this estimate is surprisingly similar to both the Deuster et al. (1999) finding among 14-44 year-olds: 8.3\% (6.4-10.2\%) and the Angst et al. (2001) estimate of $8.1 \%$ severe and $13.6 \%$ moderate (similar to our subthreshold cases) perimenstrual syndromes among 12-35-year-old community subjects. Although using slightly different symptoms we also confirm, by and large, the Angst et al. finding of a similar symptom spectrum for PMDD cases, with depressed mood, hopelessness/self depreciation, irritability/anger, affect lability, fatigability, physical complaints, anxiety/tension and the feeling of being overwhelmed as those being the most typical premenstrual problems in this age group. Another unexpected finding was, that taking into account concurrent major depression and dystyhmia and other mental disorders as DSM-IV diagnostic exclusions, the prevalence rate dropped only slightly by $0 \cdot 5-1 \%$ point. This finding might highlight a practical problem in the application of this clinical differential diagnostic issue, particularly in epidemiological research on disorders like PMDD, that refer to a duration of 12 months (i.e. the past 12 menstrual cycles). In our study we only excluded PMDD cases from the diagnosis, if there was evidence for depressive or other mental disorders over the majority of the past year, however, we assigned the PMDD diagnosis in cases where we found for example a single episode of 1 to 3 months duration only. This is in accordance with the DSM-IV stipulation, that only cases where the symptoms are merely an exacerbation should be excluded.

\section{PMDD is a relatively stable and enduring disorder}

In terms of the diagnostic and syndrome stability over a period of 48 months, our data also suggest that both threshold and subthreshold PMS are indeed relatively stable conditions. Among all baseline threshold cases only 5\% were fully remitted at the final follow-up, $47 \%$ had a least some symptoms, $31 \%$ were subthreshold and over $17 \%$ still had the full disorder. Similar proportions were found for subthreshold PMDD cases. On the other hand, only few of baseline non-PMDD cases developed into the full disorder (1\%). In accordance with a study by Rosen et al. (1990) and our incidence findings of only $2.5 \%$ incident cases (unaffected at baseline), this suggests that PMDD among young adults is a disorder that starts predominantly early in adolescence and is quite likely to persist over many years.

\section{PMDD is frequently associated with other disorders}

Our finding of high co-morbidity rates with a relatively broad spectrum of other mental disorders was only partially unexpected, given the lack of similar reports. Given the fact that PMDD in DSM-IV is grouped under depressive disorders as well as in light of some depressionlike symptoms in its criteria list, the significant association with depressive disorders (major depression and dysthymia) was expected. However, the relatively high number of bipolar I and II disorders (about 10\%) is remarkable for two reasons: first, there are some indications from the retrospective reports on age of onset that, unlike depressive disorders, which were predominantly of a secondary nature, the majority of PMDD cases had significant hypomanic symptoms prior to baseline. Similarly, the strong associations with anxiety disorders (47.4\% PMDD v. $24 \cdot 9 \%$ among non-PMDD cases), due to associations with temporally preceding social and specific phobia, particularly of blood injury and situational type, as well as with posttraumatic stress disorder (PTSD), suggest that the development of PMDD might be strongly linked to preceding anxiety disorders as well as traumatic events and PTSD. It is also noteworthy that it is very unlikely that the association to anxiety disorder is simply due to the fact that anxiety/tension is also a symptom criterion of PMDD. Clearly this link to anxiety disorder is in need of further studies, that should in particular highlight the unexpectedly strong association with PTSD as well; we are unaware of any study or clinical observation at this point that has highlighted the relevance of trauma, PTSD and PMDDD development. Given that our PTSD finding builds partly on retrospective data, which are subject to recall failure, we are unable to go beyond this observation. (But the fact that the majority of triggering traumatic 
events leading to PTSD as well as the predominant onset of anxiety before age of first menstruation lends support for this speculation.) Further indirect evidence comes from our analyses of incident co-morbid conditions. This evidence suggests that after the onset of PMDD, cases reveal a decreased risk for further incident anxiety disorders as well as no elevated risk for PTSD and traumatic events. This finding is unlike to our result for the onset of depressive and somatoform disorder. For both groups of disorders we found considerable increases in incidence after onset of PMDD.

Two other impressive associations already mentioned in the literature are with nicotine dependence (Deuster et al. 1999) and somatoform disorders. Almost $40 \%$ of all baseline PMDD cases and $20 \%$ of the subthreshold cases were regular smokers with a nicotine dependence syndrome. Although there is some decrease of the rate of dependent smokers in both groups at the end of the follow-up period, this association nevertheless stays impressively high and significant over the rates of nonPMDD sufferers. Given the assumed negative effects of smoking on PMS symptoms it is hard to speculate about the nature and the potential causal pathways involved. Clearly more research is needed to clarify whether regular smoking among adolescents increases the likelihood of PMDD; or, whether smoking has to be regarded as a dysfunctional coping reaction to the PMS problems; or, whether this association is mediated by a third vulnerability constellation.

Concerning somatoform disorders our study confirms significant associations particularly with two types: persistent pain syndromes (most frequently: dysmenorrhoea, abdominal and headache-related) and the shortened somatoform index SSI 4/6 (Escobar et al. 1989) as a subthreshold variation of somatization andless frequently - of somatization disorder. Since no sufficiently detailed analyses about age of onset of premenstrual problems and these somatoform conditions are available, we are unable to decide with sufficient precision whether premenstrual symptoms trigger the onset of somatoform disorders or vice versa, likewise our assessments were not detailed enough to study the course and the frequency of perimenstrual pains. Our longitudinal analyses, however clearly signal that PMDD leads to an increased risk of secondary pain and somatization. At baseline only $4.9 \%$ of PMDD sufferers had pain syndromes and $9.7 \%$ somatization; at final follow-up the 12 -month rates were $23.5 \%$ and $11 \cdot 6 \%$. Both conditions thus account for the observed follow-up incidence of somatoform disorders and conditions.

\section{Correlates of PMDD}

Although all PMDD cases in our sample of adolescents and young adults reported having had significant interference in several social role areas during the majority of their menstrual cycles because of their symptoms, it is noteworthy that cross-sectionally (past 4 weeks) the majority of PMDD cases were only mildly impaired and few acutely disabled. Since our study provides only 4 weeks impairment and disability measures for this cyclic monthly recurring disorder, and since most respondents were not in an acute phase of their disorder, these estimates are likely to only provide lower bound estimates of the actual impairment and disability during the acute phase.

One unexpected and previously (to our knowledge) not described finding is the high rate of PMDD sufferers reporting suicide attempts. Fifteen per cent reported at least one suicide attempt during their lifetime. This high rate could not be explained entirely by the association with major depressive episode and remained significant after controlling for the occurrence of major depression. Yet, at the same time, the risk for suicidal ideation was not significantly elevated. This finding suggests that among young PMDD sufferers there might be a group of particularly suicidal women. Further analyses will attempt to describe the profile of these women more comprehensively.

Concerning public health implications, our findings clearly demonstrate that threshold and subthreshold PMDD sufferers are high utilizers of general health, mental health and medical speciality services. In each of these categories, PMDD sufferers were much more likely to use health services than both people not affected by PMDD and the general population as the whole. It is remarkable, though, that PMDD cases: $(a)$ do not use significantly more prescribed psychotropic medications or over the counter (OTC) 
drugs, in particular, vitamins, minerals and tonics; and that $(b)$ almost $30 \%$ have been in contact with psychologists and psychotherapists, but rarely with psychiatric services. Concerning the use of OTC medications, however, it should be taken into account that we exclusively asked for the current (past 4 weeks) use of such medications. In subsequent analyses we will particularly focus on PMDD sufferers health service utilization behaviour to identify the preferred interventions as well as self-perceived improvement as a result of the use of such services.

To summarize, this community survey underlines that PMDD is a remarkably complex and clinically significant condition in adolescent and young women, leading to prolonged and quite stable suffering over many years.

This work is part of the Early Developmental Stages of Psychopathology (EDSP) Study and is funded by the German Ministry of Research and Technology, project no. 01 EB 9405/6. Principal investigators are Dr Hans-Ulrich Wittchen and Dr Roselind Lieb. Current or former staff members of the EDSP group are Dr Kirsten von Sydow, Dr Gabriele Lachner, Dr Axel Perkonigg, Dr Peter Schuster, Dr Franz Gander, Dipl.-Stat. Michael Höfler and Dipl.-Psych. Holger Sonntag as well as Mag. phil. Esther Beloch, Mag. rer. nat. Martina Fuetsch, Dipl.-Psych. Elzbieta Garczynski, Dipl.-Psych. Alexandra Holly, Dipl.Psych. Barbara Isensee, Dipl.-Psych. Marianne Mastaler, Dr Chris Nelson, Dipl.-Inf. Hildegard Pfister, Dr Victoria Reed, Dipl.-Psych. Dilek Türk, Dipl.-Psych. Antonia Vossen, Dr Ursula Wunderlich and Dipl.-Psych. Petra Zimmermann. Scientific advisors are Dr Jules Angst (Zurich), Dr Jürgen Margraf (Basel), Dr Günther Esser (Mannheim), Dr Kathleen Merikangas (Yale, New Haven) and Dr Ron Kessler (Harvard, Boston).

The authors wish to acknowledge the many helpful comments and the considerable help in revising the manuscript by Kim Yonkers, Meir Steiner and Josep Zohar.

\section{REFERENCES}

Andersch, B., Wendestam, C., Hahn, L. \& Ohman, R. (1986). Perimenstrual complaints I. Prevalence of premenstrual symptoms in a Swedish population. Journal of Psychosomatic Obstetrics and Gynaecology 5, 39-50.

American Psychiatric Association (1987). Diagnostic and Statistical Manual of Mental Disorders, 3rdedn. Revised. American Psychiatric Association: Washington, DC.
American Psychiatric Association (1994). Diagnostic and Statistical Manual of Mental Disorders, 4th ed. American Psychiatric Association: Washington, DC.

Angst, J., Sellaro, R., Merikangas, K. R. \& Endicott, J. (2001). The epidemiology of perimenstrual psychological symptoms. Acta Psychiatrica Scandinavica 104, 110-116.

Bergsjo, P., Bakket, T. \& Salamonsoen, L. (1975). Observer error in ultrasonic cephalometry. Acta Obstetrica et Gynecologica Scandinavica 54, 41-44.

Boyle, A., Berkkowitz, G. S. \& Kelsey, J. L. (1987). Epidemiology of premenstrual symptoms. American Journal of Public Health 77, 349-350.

Cleckner-Smith, C. S., Doughty, A. S. \& Grossman, J. A. (1998). Premenstrual symptoms. Prevalence and severity in an adolescent sample. Journal of Adolescent Health 22, 403-408.

Deuster, P. A., Adera, T. \& South-Paul, J. (1999). Biological, social, and behavioral factors associated with premenstrual syndrome. Archives of Family Medicine 8, 122-128.

Escobar, J. I., Rubio-Stipec, M., Canino, G. \& Karno, M. (1989). Somatic symptom index (SSI): a new and abridged somatization construct. Journal of Nervous and Mental Disease 177, 140-146.

Frank, R. T. (1931). The hormonal cause of premenstrual tension. Archives of Neurology and Psychiatry 26, 61-66.

Halbreich, U. \& Endicott, J. (1985). Relationship of dysphoric premenstrual changes to depressive disorder. Acta Psychiatrica Scandinavica 71, 331-338.

Hurt, S. W., Schnurr, P. P., Severino, S., Freeman, E. E., Gise, L. H., Riveratovar, A. \& Steege, J. F. (1992). Late luteal phase dysphoric disorder in 670 women evaluated for premenstrual complaints. American Journal of Psychiatry 149, 525-530.

Johnson, S. R. (1987). The epidemiology and social impact of premenstrual symptoms. Clinical Obstetrics and Gynecology 30, 369-384.

Johnson, S. R., McChesney, C. \& Bean, J. A. (1988). Epidemiology of premenstrual symptoms in a nonclinical sample, I: prevalence, natural history and help-seeking behavior. Journal of Reproductive Medicine 33, 340-346.

Lieb, R., Isensee, B., von Sydow, K. \& Wittchen, H.-U. (2000). The Early Developmental Stages of Psychopathology Study (EDSP): a methodological update. European Addiction Research 6, 170-182.

Logue, C. M. \& Moos, R. H. (1986). Perimenstrual symptoms: prevalence and risk factors. Psychosomatic Medicine 48, 388-414.

Merikangas, K. R., Foeldenyi, M. \& Angst, J. (1993). The Zurich study, XIX: patterns of menstrual disturbance in the community: results of the Zurich Cohort Study. European Archives of Psychiatry and Clinical Neurosciences 243, 23-32.

Monagle, L., Dan, A., Krogh, V., Jossa, F., Farinaro, E. \& Trevisan, M. (1993). Perimenstrual symptom prevalence rates: an ItalianAmerican comparison. American Journal of Epidemiology 138, 1070-1081.

Reed, V., Gander, F., Pfister, H. \& Wittchen, H.-U. (1998). To what degree does the CIDI correctly identifies DSM-IV disorders? Testing validity issues in a clinical sample. International Journal of Methods in Psychiatric Research 7, 142-155.

Rosen, L. N., Moghadam, L. Z. \& Endicott, J. (1990). Relationship between premenstrual symptoms and general well-being. Psychosomatics 31, 47-54.

Van Keep, P. A. \& Lehert, P. (1981). The premenstrual syndrome: an epidemiological and statistical exercise. In The Premenstrual Syndrome (ed. P. A. van Keep and W. H. Utian), pp. 31-42. MTP Press: Lancaster.

Widholm, O. \& Kantero, R. L. (1971). A statistical analysis of the menstrual patterns of 8000 finnish girls and their mothers. Acta Obstetrica et Gynecologica Scandinavica 3, (suppl.).

Wittchen, H.-U. (1994). Reliability and validity studies of the WHOComposite International Diagnostic Interview (CIDI): a critical review. Journal of Psychiatric Research 28, 57-84.

Wittchen, H.-U. \& Pfister, H. (eds.) (1997). DIA-X-CIDI: -Manual, Lifetime- and 12-Month Interview Version, Computer Algorithms. Swets \& Zeitlinger: Frankfurt. 
Wittchen, H.-U., Nelcon, C. B. \& Lachner, G. (1998). Prevalence and mental disorders and psychosocial impairments in adolescents and young adults. Psychological Medicine 28, 109-126.

Woods, N. F., Most, A. \& Dery, G. K. (1982a). Prevalence of perimenstrual symptoms. American Journal of Public Health $\mathbf{7 2}$, $1257-1264$.

Woods, N. F., Most, A. \& Dery, G. K. (1982b). Estimating perimenstrual distress - a comparison of two methods. Research in Nursing and Health 5, 81-91.

World Health Organization (1990). Composite International Diagnostic Interview (CIDI). WHO: Geneva.

Yonkers, K. A. (1997). Anxiety symptoms and anxiety disorders: now are they related to premenstrual disorders? Journal of Clinical Psychiatry 58 (suppl. 3), 62-67. 